\title{
Stigma in Family Patients Who Have a Psychiatric Disorder: A Systematic Review
}

\author{
Antonia Rensiana Reong and Retno Puji Astuti \\ Faculty of Nursing, Universitas Airlangga, Surabaya, Indonesia
}

\begin{abstract}
Introduction: The stigma in families who are caring for patients who experience mental illness in the modern era is high. Some of the experiences of patients with mental illness reveal that they are still stigmatized. This is not just the patients but also the families that care for them. The aim of this study was to conduct a systematic review relating to the stigma felt by the families of the patients experiencing mental illness.
\end{abstract}

Methods: The database used to identify the potential articles was Scopus. The articles were limited to a range of 5 years from 2014 through to 2018. The keywords used in the literature review were "Stigma Family" And "Mental illness".

Results: Fifteen articles from eight countries were evaluated. The families were confirmed as experiencing stigma: labeling, stereotyping, separation and discrimination. Stigma means that the families face psychological, social and intrapersonal consequences which can reduce the family quality of life and family functioning.

Conclusion: There are opportunities for negative outcomes related to the family resilience. One of the results of the study revealed that psychoeducation programs using education interventions can be an alternative intervention to reduce stigma.

\section{ARTICLE HISTORY}

Received: Dec 26, 2019

Accepted: Dec 31, 2019

\section{KEYWORDS}

stigma; family; psychiatric disorder

\section{CONTACT}

Antonia Rensiana Reong

$\triangle$ antonia.rensiana.reong-

2018@fkp.unair.ac.id

$\doteq$ Faculty of Nursing, Universitas

Airlangga, Surabaya, Indonesia

Cite this as: Reong, A. R., \& Astuti, R. P. (2019). Stigma in Family Patients Who Have a Psychiatric Disorder: A Systematic Review.Jurnal Ners, 14(3si), 86-90. doi:http://dx.doi.org/10.20473/in.v14i3(si).16992

\section{INTRODUCTION}

Schizophrenia is one type of mental health disorder that is still a complicated problem. The prevalence of severe mental illnesses, including schizophrenia, based on Riskesdas 2013 is present in 1.7 per 1000 people, which means that more than 400,000 people suffer from severe mental disorders in Indonesia. The incidence of schizophrenia is difficult to decrease due to the high recurrence rates. The recurrence rate of schizophrenia patients in Indonesia was $50 \%$ e $80 \%$, $57 \%$ within 3 years and $70 \%$ e82\% over the first five year (Rizki Fitryasari, Yusuf, Nursalam, Tristiana, \& Nihayati, 2018). Mental health stigma may have a detrimental effect on people due to their hesitation to disclose their psychiatric diagnoses, as well as decreased help-seeking attitudes and treatment compliance, potentially worsening prognosis (Ndetei et al., 2015). Stigma has been linked to adversity for people with a mental disorder as it acts as a barrier to help-seeking and it can affect all aspects of a person's life. Stigma is referred to as a social construct comprised of four interrelated components that include: 1) people distinguish and label human differences as a knowledge problem; 2) labeled people incur damage through negative stereotypes as an attitude problem; 3) labeled people are placed in exclusion; and 4) labeled people lost their status and are discriminated against.

A stigmatized attitude was related to the reason people consider mental disorder itself. The stigma of mental health has a detrimental effect on people because they tend to hesitant to reveal a psychiatric diagnosis, display a decrease in mental healthseeking attitude, and a decreased medication adherence potentially worsens the disease prognosis. Stigma and discrimination related to mental health not only affects the people living with schizophrenia but also their entire family. Reducing stigma and discrimination is the key to responding to the unmet needs of people with mental illness. Local contexts are very important in this effort because their stigma 
and manifestations depends on the specific conditions of the target population and across cultures and settings (Krupchanka et al., 2018).

Stigma modulates what is revealed in the disclosure of depression, the life experiences of people who experience psychiatric disorders after the disclosure, and how striving for health services becomes stigmatized (Brijnath \& Antoniades, 2018). Stigma basically hides the mental illness from the community and puts pressure on the families to provide support who may not have the expert knowledge needed by the clinical consumers. The caring and continuous nature of caring can cause the caregivers to develop stigmatic attitudes and attitudes (Sawrikar \& Muir, 2018). Shame focuses on stigma as a form of social responsibility and it is a socio-psychological consequence of mental illness (Subandi \& Good, 2018).

Affiliate stigma often refers to the stigma that is internalized among the family members of stigmatized individuals (Zhang et al., 2018). The stigma associated with mental illness, which permeated the context of Chinese culture, had a good effect on people with schizophrenia and their family caregivers (Wong, Kong, Tu, \& Frasso, 2018). Internalized stigma is a barrier to mental health care in China, and stigma reduction is expected to encourage the use of treatment, especially for mood disorders and schizophrenia (Smith et al., 2018). Some people with mental illness in China do not receive treatment. This proves how stigma and family obligations affect the accessibility of social support for patients with depression in China and the potential acceptance of peer support programs (Yu, Kowitt, Fisher, \& Li, 2018). Generally, people blame the family members for the development of their mental illness and they may expect them to assume the responsibility of caring for their sick relatives. For example, parents can be accused of causing illness; siblings and spouses are blamed for ignorance (Chang et al., 2018).

The inability of the family to control emotions, and the existence of stressful family life problems, means that continuous criticism from the family is the cause of the recurrence of patients with mental disorders. This phenomenon shows that the family has not been able to care for the family members who have schizophrenia. There is the inability to be influenced by various stressors. Stressors involve many types of care burden, negative perceptions or stigma and a lack of support from the surrounding environment. The stressors experienced by the families may be mediated by resilience, the family's ability to survive and rise to determine what they will do, and the ability to care for family members who suffer from schizophrenia (R. Fitryasari, Yusuf, Nursalam, Tristiana, \& Nihayati, 2018).

Family plays an important role in the way that an individual manages the emotions of psychiatric disorders experienced by individuals. Families play a role in providing social support through family communication (Flood-Grady \& Koenig Kellas, 2018).
Family communication around the sufferers suffering from mental illness is not uniformly positive. Family interactions precede, and sometimes worsen, the members' experiences with mental illness, and their relationship with members who experience psychiatric disorders can expose them to stigma (Flood-grady, Kellas, \& Flood-grady, 2018).

The families of people with mental illness are considered to be important resources rather than as contributors to their mental illness. However, these families not only experience the burden of care but also the social stigma and discrimination in various aspects of their lives. Their psychosocial needs tend to be neglected (Hyun, Nam, \& Kim, 2018). One study showed that one of the interventions to reduce stigma, namely using the family psycho-education program, showed a reduction in post-intervention stigma by using contact interventions and educational interventions which had a small-medium direct effect on stigma (Morgan, Reavley, Ross, Too, \& Jorm, 2018). The purpose of this systematic review must be considered to reduce and prevent stigma in clients with mental disorders.

\section{MATERIALS AND METHODS}

The database search focused on the Scopus online journal database and the publication media therein. The keywords included were "Family Stigma" and "Mental illness"; 989 related articles were found. From the related articles, 15 articles were taken that met the inclusion criteria. The articles included in the summary of this literature were (1) articles published in Scopus indexed journals, (2) articles focusing on the assessment of stigma in adults or children whose children are concerned with health and change, (3) quantitative or qualitative research methods related to stigma in general, self-stigma, and stigma on the families, (4) articles within the last 5 years from 2014-2018 and (5) articles written in English. The initial Search Results totaled 989 articles, abstracts and the full texts $n=55$ Articles. The issuance of the selected articles (Main article selected) after the abstract titles and collections $n=934$ Articles, Articles that do not comply with the inclusion criteria $n=40$ Articles; 34 were entered under the general stigma in 6 criteria, focused on the stigma of mental illness. Publication according to the inclusion criteria articles $\mathrm{n}=15$ articles, while the publications that included systematic reviews n-15.

\section{RESULTS}

Stigma in people who experience psychiatric disorders is not only attached to the sufferer but to their whole family. This sometimes becomes an obstacle to improving the quality of life of people who experience psychiatric disorders, whereas we know that families play an important role as a support system for their quality of life. Stigma and emotional expression are the factors that exacerbate social interaction in both the family and society, in the form of family communication in meeting the psychological 
needs of the patients and their families as support systems through interaction (Khoirunnisa, Syuhaimie Hamid, \& Catharina Daulima, 2018). Patients with mental illness report barriers that prevent them from (a) receiving treatment and (b) relying on informal social support from family members, including stigma, somatization, and community norms. Peer support (support from others with depression) is seen by the patients as an acceptable means of exchanging information and relying on others for support (Yu et al., 2018). In this literature study, the family experiences of the patients experiencing psychiatric disorders will be discussed.

The burden of care felt by the family is related to confusion about the illness, emotional burden, physical problems, time constraints, and financial and social problems, which cause a decrease in the quality of family life. Families also experience stigma through labeling, stereotyping, separation and discrimination. Stigma means that families face psychological, social and intrapersonal consequences. This decreases the family quality of life and family functioning, and there are opportunities for negative outcomes related to family resilience. Health workers, especially psychiatric nurses, must review the burden of care and stigma to develop nursing interventions so then the families can achieve resilience (R. Fitryasari et al., 2018). The experience of stigma of 25 different family members related to people living with schizophrenia included four levels of the respondents' lives (macro, micro, and intro-level). The overall issues were (1) a lack of general understanding and misunderstandings about mental illness; (2) structural discrimination and a lack of government and public support systems; (3) the burden of "pervasive and unlimited" care and an inability to live independently (Krupchanka et al., 2018) .

Overall, it was found that their 'stories' were very different from each other, which caused high stigma in the family. In short, the widespread stigma associated with mental illness in society affects the people from gaining in-depth knowledge about it and therefore the ability to recognize the initial symptoms and to provide the empathy needed. It also impacts on the capacity of the health professionals to provide holistic care and not only to depend on 'medical models'. Providing adequate funding to ensure that a formal mental health system has the right infrastructure to meet consumer needs. Stigma basically hides the mental illness from the community and puts pressure on the families to provide support, who may not have the expert knowledge needed by clinical consumers. The continuous and stressful nature of caring can cause the caregivers to develop stigmatic attitudes. This is intensified by the misperceptions of consumer control related to their mental illness, the uneven distribution of attention in the household, and low consumer insight into the caregiver experiences. This can also lead to the development of their own trauma which requires formal assistance. To overcome these complex problems, stigma is a major problem that requires improvement (Sawrikar \& Muir, 2018).

Most caregivers of individuals with mental illness experience affiliate stigma in Singapore. The four QOL domains are significantly related to affiliate stigma (Zhang et al., 2018). The thematic analysis of the data showed that the participants experienced shame in three different ways. First, is as a cultural index of disease and recovery. The family members identify their family member's illness when they lose their shame. If the patient shows an attitude that shows the reappearance of shame, the family sees this as an indication of recovery. Second, is as an indication of recurrence. Third, is as a barrier to recovery (Subandi \& Good, 2018). People with schizophrenia and their caregivers have both internalized negative judgments from their social networks and reduced their involvement in society. The participants with schizophrenia expressed shame and inferiority, talked about being a burden to their family, and expressed self-disappointment as a result of their psychiatric diagnosis. The caregivers reveal a high level of emotional stress due to mental illness in the family. The family figures vary as far as internal stigma experienced by patients and caregivers goes. The family plays a central role in treating people with mental illness in China (Wong et al., 2018).

\section{DISCUSSION}

Family stigma is low in the early stages of a psychotic disorder, with the families endorsing many supportive statements. For example, the patients are often encouraged to vote and the patients want to work. The family members express a belief that mental illness should be protected legally as a disability and that parity should exist in insurance coverage. One characteristic of family stigma is social isolation. The members of the families keep to themselves and they usually do not invite others into the home or tell them what is happening (Khoirunnisa et al., 2018). The implementation of family duties requires the skill and ability of all members of the family to achieve the highest degree of health. Establishing and maintaining good relationships with the patients is an important aspect of the treatment and healing process (Khoirunnisa et al., 2018). In order to better identify which aspects of the interventions are associated with effectiveness, the studies should aim to report the intervention characteristics in more detail. These include implementation characteristics (e.g. dose and fidelity) as well as the characteristics drawn from the intergroup contact theory that have been proposed as important (e.g. for contact interventions, the type of interaction and comparative status of participants and the person with lived experience; for education interventions, the type of information including experiences of recovery, stigma, and how to provide support). A greater emphasis on how interventions reduce stigma, such as the role of knowledge, anxiety and empathy, would also advance the literature. 
The results of one study on a family psychoeducation program showed a reduction in stigma after the intervention, namely by using combination interventions; contact interventions and educational interventions have a small-medium direct effect on stigma (Morgan et al., 2018). In addition, this can be utilized by peer support communication (i.e., support from fellow mental illness patients). It is seen by the patients as an acceptable means to exchange information and to support each other in the process of undergoing treatment therapy (Yu et al., 2018). In addition, a therapeutic communication approach is made in an effort to motivate the patient's family in a joint effort to reduce fear, shame, burden, and emotional stress and to develop a culture of respect and attention to people with psychiatric disorders.

\section{CONCLUSION}

The family is an internal support system that plays an important role in achieving the expected quality of life, especially in patients who experience psychiatric disorders. However, even in this modern global era, there are still many people who stigmatize not only the patients with psychiatric disorders but also their families. This is a serious obstacle to the successful improvement of the positive quality of life expected in mental illness patients because of the loss of important internal supporting factors, namely their family. This can increase the recurrence in patients; therefore, it is necessary to design an effective and efficient therapeutic communication intervention method to minimize the stigmatization in mental ilness in order to improve the quality of life of mental illness patients by involving their families. The family psychoeducation program shows a reduction in stigma after the intervention, namely by using a combination of interventions; contact interventions and educational interventions have small-medium direct effects on stigma, but further research is needed to investigate how to sustain the benefits in the long term and how to support the interventions to maximize their effectiveness (Morgan et al., 2018). In addition, peer support (support from fellow mental illness patients) is seen by the patients as an acceptable means to exchange information and to support each other in the process of undergoing treatment therapy (Yu et al., 2018).

\section{REFERENCES}

Brijnath, B., \& Antoniades, J. (2018). What is at stake? Exploring the moral experience of stigma with Indian-Australians and Anglo-Australians living with depression. Transcultural Psychiatry, 55(2), 178-197. https://doi.org/10.1177/1363461518756519

Chang, C. C., Su, J. A., Chang, K. C., Lin, C. Y., Koschorke, M., \& Thornicroft, G. (2018). Perceived stigma of caregivers: Psychometric evaluation for Devaluation of Consumer Families Scale. International Journal of Clinical and Health
Psychology, 18(2), 170-178. https://doi.org/10.1016/j.ijchp.2017.12.003

Fitryasari, R., Yusuf, A., Nursalam, Tristiana, R. D., \& Nihayati, H. E. (2018). Family members' perspective of family Resilience's risk factors in taking care of schizophrenia patients. International Journal of Nursing Sciences, 5(3). https://doi.org/10.1016/j.ijnss.2018.06.002

Fitryasari, Rizki, Yusuf, A., Nursalam, Tristiana, R. D., \& Nihayati, H. E. (2018). Family members' perspective of family Resilience's risk factors in taking care of schizophrenia patients. International Journal of Nursing Sciences, 5(3), 255-261.

https://doi.org/10.1016/j.ijnss.2018.06.002

Flood-grady, E., Kellas, J. K., \& Flood-grady, E. (2018). Sense-making , Socialization, and Stigma: Exploring Narratives Told in Families About Mental Illness Sense-making, Socialization, and Stigma: Exploring Narratives Told in Families About Mental Illness. Health Communication, o0(00), $1-11$. https://doi.org/10.1080/10410236.2018.14310 16

Flood-Grady, E., \& Koenig Kellas, J. (2018). Sensemaking, Socialization, and Stigma: Exploring Narratives Told in Families About Mental Illness. Health Communication, 00(00), 1-11. https://doi.org/10.1080/10410236.2018.14310 16

Hyun, M.-S., Nam, K. A., \& Kim, H. (2018). Effects of a brief empowerment program for families of persons with mental illness in south korea: A pilot study. Issues in Mental Health Nursing, o(0), 1-7. https://doi.org/http://dx.doi.org/10.1080/0161 2840.2018.1453000

Khoirunnisa, M. L., Syuhaimie Hamid, A. Y., \& Catharina Daulima, N. H. (2018). Family experiences in communicating with family members experiencing social isolation after hospitalization. Enfermeria Clinica, 28, 116-121. https://doi.org/10.1016/S1130-8621(18)300494

Krupchanka, D., Chrtková, D., Vítková, M., Munzel, D., Čihařová, M., Růžičková, T., ... Sartorius, N. (2018). Experience of stigma and discrimination in families of persons with schizophrenia in the Czech Republic. Social Science and Medicine, 212, 129-135. https://doi.org/10.1016/j.socscimed.2018.07.01 5

Morgan, A. J., Reavley, N. J., Ross, A., Too, L. S., \& Jorm, A. F. (2018). Interventions to reduce stigma towards people with severe mental illness: Systematic review and meta-analysis. Journal of Psychiatric Research. https://doi.org/10.1016/j.jpsychires.2018.05.01 7

Ndetei, D. M., Mutiso, V., Maraj, A., Anderson, K. K., Musyimi, C., \& Mckenzie, K. (2015). Stigmatizing attitudes toward mental illness among primary school children in Kenya. Social Psychiatry and 


\section{Psychiatric}

Epidemiology. https://doi.org/10.1007/s00127-015-1090-6

Sawrikar, P., \& Muir, K. (2018). Toward a familyoriented treatment approach for consumers and carers of mental illness. International Journal of Mental Health, 47(2), 158-183. https://doi.org/10.1080/00207411.2017.13674 48

Smith, M. L., Yang, L. H., Huang, D., Pike, K. M., Yuan, C., \& Wang, Z. (2018). Measuring internalized stigma of mental illness among Chinese outpatients with mood disorders. International Journal of Culture and Mental Health, O(0), 1-14. https://doi.org/10.1080/17542863.2018.14424 84

Subandi, M. A., \& Good, B. J. (2018). Shame as a cultural index of illness and recovery from psychotic illness in Java. Asian Journal of Psychiatry, 34(2010), 33-37. https://doi.org/10.1016/j.ajp.2018.04.005
Wong, Y. L. I., Kong, D., Tu, L., \& Frasso, R. (2018). “My bitterness is deeper than the ocean": Understanding internalized stigma from the perspectives of persons with schizophrenia and their family caregivers. International Journal of Mental Health Systems, 12(1), 1-15. https://doi.org/10.1186/s13033-018-0192-4

Yu, S., Kowitt, S. D., Fisher, E. B., \& Li, G. (2018). Mental Health in China: Stigma, Family Obligations, and the Potential of Peer Support. Community Mental Health Journal, 54(6), 757-764. https://doi.org/10.1007/s10597-017-0182-z

Zhang, Y., Subramaniam, M., Lee, S. P., Abdin, E., Sagayadevan, V., Jeyagurunathan, A., ... Chong, S. A. (2018). Affiliate stigma and its association with quality of life among caregivers of relatives with mental illness in Singapore. Psychiatry Research, 265, 55-61. https://doi.org/10.1016/j.psychres.2018.04.044 